\title{
Editorial
}

\section{Food environments: measuring, mapping, monitoring and modifying}

Increasing access to healthy and affordable foods in communities is an identified priority of national health agendas in a number of countries ${ }^{(1-3)}$. Arguably, however, understanding of the particular food access and environmental exposures most important for a healthy diet among different populations remains incomplete.

The empirical investigation of food environments important for diet and health is a relatively young but burgeoning field. Six years ago, in November 2007, the US National Cancer Institute (NCI), in partnership with the Robert Wood Johnson Foundation and other National Institutes of Health centres, convened a workshop aimed at identifying the knowledge gaps and future research needs with regard to measures of food (and physical activity) environments ${ }^{(4)}$. Key outcomes of that meeting included advocacy for refinements in conceptual models linking food environments with dietary and health outcomes; and for the advancement of methodologies and measurements of food environmental exposures, including tailoring for communities with particular need (e.g. low income, ethnic minorities). Since then, research into food environments and their impact on eating and health has expanded dramatically. Despite this, findings are far from consistent and many questions remain concerning how, why and for whom environmental factors impact on food purchasing and consumption and associated health outcomes. Efforts to advocate for environmentally focused policies and programmes to improve diet, or to counter environmental initiatives that might contribute to poor diet, have at times floundered as a result. Yet solid evidence is particularly important in this field given that environmental and policy changes are often difficult and expensive to leverage.

This issue of Public Health Nutrition includes a selection of papers reporting on investigations into various aspects of the food environment or 'foodscapes' which contribute to addressing the key conceptual and methodological needs raised at the NCI workshop. In the broader food environment literature, environments have been conceptualised at the community (e.g. the type and location of food retail facilities), consumer (e.g. the availability, price, quality of foods within a facility), organisational (e.g. food sources within schools and worksites) and information (e.g. the media and advertising) levels ${ }^{(5)}$; all four of these are considered in this issue. Across these domains, the papers can be broadly considered as assessing measurement issues ${ }^{(6,7)}$; mapping and describing food environments ${ }^{(8-10)}$; and linking environmental exposures with diet ${ }^{(11-13)}$.

\section{Measurement}

The accurate identification of environmental effects on diet requires robust measures of environmental exposures, including measures that are sensitive, specific and appropriate for particular target groups and localities. Hearst and colleagues ${ }^{(6)}$ remind us, firstly, that the home also comprises an environmental exposure in terms of food access and is in fact typically the key exposure for young children; and secondly, that the use of existing food environment measures - in this case the Home Food Inventory - for groups with particular cultural or socioeconomic needs is not always appropriate. While the inventory functioned relatively well among low-income Spanish-speaking households, it required substantial revision among Somali-speaking participants. In a vastly different population group, yet one also characterised by a high burden of disease attributable to poor nutrition, Brimblecombe and colleagues ${ }^{(7)}$ examined the feasibility of using point-of-sale data to monitor diet quality of food sales in stores in remote Aboriginal Australia. Findings, demonstrating the low cost, low burden and high feasibility of this objective form of assessment, suggest exciting potential for future applications in nutrition monitoring or intervention evaluation.

\section{Mapping}

Three papers report on studies mapping and describing food environments in varied settings - within neighbourhoods ${ }^{(8)}$, supermarkets ${ }^{(9)}$ and dining venues near educational institutions ${ }^{(10)}$. In their study, Schneider and Gruber $^{(8)}$ demonstrated a neighbourhood socioeconomic gradient in fast-food outlet density and accessibility, confirming findings of previous studies and extending these to Cologne, Germany. Commendably, given the lack of existing registries of food outlet locations, in their study the researchers sourced these themselves on foot or by bicycle. Such 'ground-truthing' overcomes potential limitations of incomplete environmental data that have been associated with reliance on secondary forms of food outlet data, such as government or commercial sources $^{(14,15)}$. Given the similar socio-economic gradients 
found in their study for alcohol and tobacco retail outlets, the authors suggest that 'addictive environments' cluster in socio-economically disadvantaged neighbourhoods (although inclusion of healthy food sources in the study would have helped to rule out the possibility that the clusters simply reflected greater commercialisation in these neighbourhoods). Similarly, Cameron and colleagues ${ }^{(9)}$ demonstrated the potential contribution of features of the food environment to the emergence or maintenance of health inequalities, reporting socio-economic gradients in exposure to energy-dense snacks and beverages within supermarkets in Melbourne, Australia. Collectively, findings of these two studies indicate a potential culmination of health risk exposures of critical concern for those engaged in addressing health inequalities. In the final ecological study, Horacek et al. ${ }^{(10)}$, like Cameron et al., ventured into food retail environments to examine within-store foodscapes - in this case, the dining environments of 243 campus dining outlets or restaurants located on or near fifteen post-secondary educational institutions. They found that unhealthful dining environments - indicated by such features as the low availability of healthy entrées/salad bar, fewer facilitators (e.g. information, healthy promotions, reduced portion sizes) and more barriers (e.g. unhealthy promotions, large portion sizes) to healthy eating - were prevalent in these locations.

\section{Association with individual diet}

While each contributes novel data on the foodscapes of various settings, the three ecological studies described above cannot provide conclusive evidence as to the impact of environmental features on diet. Moving further towards that end, two of the papers in this issue have linked environmental exposures with individual behaviour. Van Ansem et al. ${ }^{(11)}$ confirm previous reports that the home food environment - specifically, home availability of fruit and vegetables - is positively predictive of children's intakes of these foods. However, they advance previous work by investigating potential determinants of home availability of fruits and vegetables. While their candidate determinants - parental perceptions of local food environments - were not strongly predictive of home availability of fruits and vegetables, their study raises for future research to consider the important question of what it is that leads some parents to purchase and make available these foods within their homes. That study also raises the issue of the importance of considering subjectively assessed environmental exposures. Subjective reports of food environments do not always match those obtained via objective assessment ${ }^{(16)}$ and they may not predict dietary outcomes in the same way ${ }^{(17)}$. Nevertheless, if subjective food environmental perceptions do predict intakes, they may serve as important intervention levers, regardless of their 'accuracy' when considered alongside objective measures.

The study by Jack and colleagues ${ }^{(11)}$ found that food outlet density did not predict fruit or vegetable consumption, reminding us that the evidence linking diet with healthy food access is not consistent. However, their study assessed access to food outlets at postcode level only. Assessment of food outlets within an administratively defined region, and near home locations only, is not uncommon among studies in this field. However, this approach does not take into account exposures beyond the residential neighbourhood that might influence food choices, and hence it may mischaracterise the nature of environmental influences on diet. Capturing all potential food-related environmental features, including nonresidential exposures (e.g. food stores near work location, recreational or social activities, or child care) is possible through the investigation of activity spaces. Assessment of an activity space - a concept derived from behavioural geography that refers to all destinations visited within a specified time period and the travel routes used $^{(18)}$ - provides a more comprehensive picture of the multiple contexts to which people are regularly exposed. Early findings ${ }^{(19)}$ suggest this approach may identify environmental determinants of diet that are erroneously missed in investigations relying on more typically used residential characterisations of neighbourhood. An activity space approach represents a key methodological advance with much potential for future studies of place effects on diet and health and for informing intervention and policy development.

Finally, the review of the effects of subsidies on healthy food purchasing and consumption ${ }^{(13)}$ provides relatively conclusive evidence about the impact of one key feature of the food environment - pricing - on food purchasing and consumption behaviours. Of twenty distinct field experiments, An reports that all but one found subsidies on healthy foods to significantly increase purchasing of those foods. While a number of questions relating, for example, to dose-response and cost-effectiveness remain, these findings add to a now relatively solid accumulated body of evidence confirming the effectiveness of economic incentives in modifying dietary behaviours. The author laments, however, that in spite of such evidence, policy adoptions remain scarce.

\section{Research needs}

What does this collective of papers, considered in the context of the broader food environment literature, tell us about future research needs? In addition to the research gaps raised above, the field could benefit from continued efforts to accurately measure and monitor environmental exposures; more evidence linking exposures with diet in different populations and contexts; and a better 
understanding of how food environments influence diet and for whom (for example, research on mediators and moderators of environment-diet associations).

There is also a need to remain mindful of abundant existing evidence of other determinants of dietary intake. We should not overlook decades of research testifying to the importance of key personal, social and other contextual determinants of diet. Is the establishment of a major food retail outlet likely to have any impact on diet in a neighbourhood where the majority of residents have few relevant food procurement or cooking skills, lack motivation to change, perceive healthy food as unaffordable and 'for the wealthy', and are exposed to social norms that embrace fast-food consumption over home-cooked vegetables? Such factors are particularly important to consider in environments where unhealthy options are abundant and removal or replacement of these with healthier alternatives is unlikely ${ }^{(20)}$. Researchers are well advised to consider and integrate theoretically grounded determinants from all levels of influence to inform future investigations and generate a more comprehensive understanding of the complex array of both proximal and distal influences on diet.

In cross-sectional studies, non-random residential selection precludes inferences that neighbourhood environments directly influence eating behaviours, and logistics and ethics make it difficult - at times impossible - to test this. While some researchers have attempted to use analytic approaches to deal with issues of residential selection ${ }^{(21)}$, solid evidence that neighbourhood food exposures are causally associated with diet is lacking. But is this important? Schneider and Gruber ${ }^{(8)}$ argue that the cause of associations of environments with diet is not as important for public health initiatives as the resulting consequences regarding neighbourhood environments and health. It may not matter, for example, to know whether food supply follows or creates demand, if the health consequences for the residents are the same.

\section{Public health imperatives}

What can be done to address these consequences? In terms of advocacy, practice and policy implications, the papers in this issue and the broader literature suggest a number of imperatives. Efforts are required to combat exposure to foodscapes that are inconsistent with recommendations for healthy eating, as well as food environments that may maintain socio-economic inequalities in nutrition-related outcomes. Actions towards this could include urban planning policies encouraging retail mix and diverse food outlets; licensing laws and restrictions on food outlet location/density; or increasing permeability/ travel between neighbourhoods by investing in public transport.

Is the evidence strong enough yet to advocate for change? Increasingly the body of evidence is building a case that the food environment does matter, although, as described above, there remain gaps and inconsistencies that hopefully future research will address. In some cases, however, such as in the case of the positive effective of pricing incentives ${ }^{(13)}$, we have accumulated sufficient evidence to act. In these instances, environmental and policy change to modify food environments should be complemented with rigorous evaluation of dietary, health and economic impacts to continue to build the evidence base and facilitate future public health action to foster healthy food access for all.

\section{Acknowledgements}

K.B. is supported by a National Health and Medical Research Council Principal Research Fellowship, ID 1042442. L.T. is supported by a Deakin University Alfred Deakin Postdoctoral Research Fellowship.

$$
\text { Kylie Ball }{ }^{1,2} \text { and Lukar Thornton }{ }^{2}
$$

$$
\begin{array}{r}
{ }^{1} \text { Associate Editor } \\
\text { Email: kylie.ball@deakin.edu.au }
\end{array}
$$

${ }^{2}$ School of Exercise and Nutrition Sciences

Deakin University

221 Burwood Highway, Burwood, VIC 3125, Australia

Email: lukar.thornton@deakin.edu.au

\section{References}

1. National Prevention Council (2011) National Prevention Strategy. Washington, DC: US Department of Health \& Human Services, Office of the Surgeon General; available at http://www.healthcare.gov/prevention/nphpphc/strategy/ report.pdf

2. HM Government (2010) White Paper: Healthy Lives, Healthy People: Our Strategy for Public Health in England. London: HM Government; available at https://www.gov. uk/government/publications/healthy-lives-healthy-peopleour-strategy-for-public-health-in-england

3. Australian National Preventative Health Taskforce (2009) Australia: The Healthiest Country by 2020. National Preventative Health Strategy - The Roadmap for Action. Canberra: Commonwealth of Australia; available at http:// www.preventativehealth.org.au/internet/preventativehealth/ publishing.nsf/Content/CCD7323311E358BECA2575FD000859E1/ $\$$ File/nphs-roadmap.pdf

4. McKinnon RA, Reedy J, Handy SL et al. (2009) Measuring the food and physical activity environments: shaping the research agenda. Am J Prev Med 36, 4 Suppl., S81-S85.

5. Glanz K, Sallis JF, Saelens BE et al. (2005) Healthy nutrition environments: concepts and measures. Am J Health Promot 19, 330-333.

6. Hearst MO, Fulkerson JA, Parke M et al. (2013) Validation of a home food inventory among low-income Spanishand Somali-speaking families. Public Health Nutr 16, 1151-1158.

7. Brimblecombe J, Liddle R \& O'Dea K (2013) Use of pointof-sale data to assess food and nutrient quality in remote stores. Public Health Nutr 16, 1159-1167. 
8. Schneider S \& Gruber J (2013) Neighbourhood deprivation and outlet density for tobacco, alcohol and fast food: first hints of obesogenic and addictive environments in Germany. Public Health Nutr 16, 1168-1177.

9. Cameron AJ, Thornton LE, McNaughton SA et al. (2013) Variation in supermarket exposure to energy-sense snack foods by socio-economic position. Public Health Nutr 16, 1178-1185.

10. Horacek TM, Erdman MB, Byrd-Bredbenner C et al. (2013) Assessment of the dining environment on and near the campuses of fifteen post-secondary institutions. Public Health Nutr 16, 1186-1196.

11. Jack D, Neckerman K, Schwartz-Soicher O et al. (2013) Socio-economic status, neighbourhood food environments and consumption of fruits and vegetables in New York City. Public Health Nutr 16, 1197-1205.

12. Van Ansem WJB, Schrijvers CTM, Rodenburg G et al. (2013) Is there an association between the home food environment, the local food shopping environment and children's fruit and vegetable intake? Results from the Dutch INPACT study. Public Health Nutr 16, 1206-1214.

13. An R (2013) Effectiveness of subsidies in promoting healthy food purchases and consumption: a review of field experiments. Public Health Nutr 16, 1215-1228.

14. Lake AA, Burgoine T, Greenhalgh F et al. (2010) The foodscape: classification and field validation of secondary data sources. Health Place 16, 666-673.
15. Liese AD, Colabianchi N, Lamichhane AP et al. (2010) Validation of 3 food outlet databases: completeness and geospatial accuracy in rural and urban food environments. Am J Epidemiol 172, 1324-1333.

16. Williams LK, Thornton L, Crawford D et al. (2012) Is the objective food environment associated with perceptions of the food environment? Public Health Nutr 15, 291-298.

17. Giskes K, van Lenthe FJ, Brug J et al. (2007) Socioeconomic inequalities in food purchasing: the contribution of respondent-perceived and actual (objectively measured) price and availability of foods. Prev Med 45, 41-48.

18. Thornton LE, Pearce JR \& Kavanagh AM (2011) Using Geographic Information Systems (GIS) to assess the role of the built environment in influencing obesity: a glossary. Int J Behav Nutr Phys Act 8, 71.

19. Zenk SN, Schulz AJ, Matthews SA et al. (2011) Activity space environment and dietary and physical activity behaviors: a pilot study. Health Place 17, 1150-1161.

20. Thornton LE, Jeffery RW \& Crawford D (2012) Barriers to avoiding fast-food consumption in an environment supportive of unhealthy eating. Public Health Nutr (Epublication ahead of print version).

21. Zick CD, Hanson H, Fan JX et al. (2013) Re-visiting the relationship between neighbourhood environment and BMI: an instrumental variables approach to correcting for residential selection bias. Int J Behav Nutr Phys Act 10, 27. 